\title{
A Routing Algorithm via Constructing the Weighted Minimum Connected Dominating Set in Mobile Ad- hoc Network
}

\author{
Zifen Yang ${ }^{12}$ \\ Linyi University \\ Linyi, 276000,china \\ E-mail: yangzifenelyu.edu.cn
}

\author{
Guoqiang $\mathbf{L i}^{3 a}$, Guangquan $\mathbf{F u}^{\mathbf{b}}$ \\ Linyi University \\ Linyi, 276000, china \\ E-mail: " I i guoqiangl Iyu. edu. cn ; fuguangquand lyu.edu. cn
}

\section{WenPeng Lu}

QiLu University Of Technology

Jinan, 250353, china

E-mail: I wp@qlu.edu.cn

\begin{abstract}
A mobile ad-hoc network (MANET) is made up of a group of mobile wireless nodes, which can be flexibly and quickly applied to many applications. The devices in MANET are often suffering from the issue of battery power since they cannot be charged in time. Then, as benefitting from the fact that the fewer nodes provide data forwarding support and the less energy will be consumed, many methods utilize the connecting dominate set (CDS) to prolong the network lifetime. However, constructing a smallest size of CDS is a NP-complete problem. In this paper, we propose a new greedy approach constructing a weighted CDS (Cgreedy-wMCDS) by integrating three factors such as energy, mobility and degree of nodes. The proposed method provides the whole network control and data forwarding support. This new method incorporats route stability into the routing algorithm to reduce the route path failures. The simulation results show that the approach of Cgreedy-wMCDS is superior to other methods in terms of prolonging the lifetime of MANET. The time complexity of our algorithm is $O(D)$, where $D$ is the network diameter.
\end{abstract}

CENet2017

22-23 July 2017

Shanghai, China

\footnotetext{
${ }^{1}$ Speaker

${ }^{2}$ This work was supported by Shandong Provincial Natural Science Foundation, China (ZR2014FL013), PhD research foundation of Linyi University (LYDX2016BS077), and partlysupported by Natural Science Foundation, China(NSF: 61502259).

${ }^{3}$ Corresponding Author
} 


\section{Introduction}

Mobile ad-hoc network (MANET)combines mobile communication and computer network which consists of wireless nodes, which do not rely on a predefined infrastructure [1] but can provide an instant and distributed peer-to-peer ad hoc communication [2]. In a MANET, two nods can communicate directly with each other if they are within each other's transmission range. At meanwhile, the intermediate nods have to route message for two nodes when they are not in each other's transmission range. Hence, an intuitive choice is to introduce a strategy of multi-hop routing in MANET [3]. Each node acts as a routing node responseding to the changes of network topology [4]. A popular routing protocol for MANET utilizes a broadcasting scheme to discover a rout, in which the nodes will repeat sending orreceiving broadcast signal [5]; however, the flooding of signal causes congestion and consumes more battery power which is impossible to get recharged in time.

As benefitting from the fact that the fewer nodes provide data forwarding support, the less energy will be consumed, the connecting dominate set (CDS) as the backbone is an effective strategy to prolong the network lifetime.A CDS for a graph $G(V, E)$ is a subset $V^{\prime}$ of $V$, and each node in $V-V^{\prime}$ is adjacent to some node in $V^{\prime}$, which induces a connected subgraph. For the routing based on CDS, there are many advantages, for example, the search space is restricted [6], flooding storm can be avoided, the routing overhead can be reduced, and less energy can be consumed and the lifetime of MANET can be prolonged. In other words, MANET, based on CDS, can reduce the communication overhead, increase the convergence speed and simplify connectivity management. Thus, how to construct the minimum size connected dominating set (MCDS) of the network becomes a desirable aim. Due to the energy issue of MANET, the algorithm should not only construct thinner CDS but also construct with low computation and communication costs.

Based on the above considerations, we propose a novel approach to construct a weighted CDS (Cgreedy-wMCDS) by integrating three factors: energy, mobility and degree.

We list main contributions of this work as follows:

a) The proposed approach takes three factors: energy, mobility and degree into consideration.

b) The time complexity of our algorithm is $O(D)$, where $D$ is defined to the network diameter.

c) The new approach is a distributed algorithm, which does not need to know the entire network topology information, but only depends on the information of two-hop neighbor information.

\section{Related Work}

\subsection{Related Network Modeling and Notations}

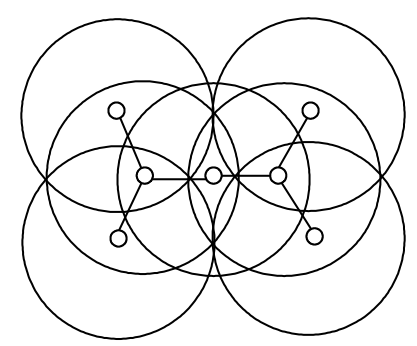

Figure 1: Topology of Wireless ad Hoc Networks by Unit-disk Graphs 
The paper assumes that all MANET nodes in $V$ are distributed in a two-dimensional plane and have an equal maximum transmission range of one unit, as illustrated in Figure 1. That is to say, two nodes $u$ and $v$ are neighbors if the euclidean distance between them is at most unity. Each node has the same communication radius.

Some notations are depicted as follows:

$N(u): \ldots \ldots \ldots$...Neighbor set for node $u E_{r m}(u): \ldots \ldots .$. The remaining energy of node $u$

$E_{i n}(u) \ldots \ldots . . .$. .Initial energy of node $u S T(u) \ldots \ldots .$. The current state of node $u$

Using a CDS as a virtual backbone for routing was proposed in Paper [7]. Since then, many works have been carried out to design approximations algorithms for CDS construction. Those algorithms can be divided into centralizedand distributed algorithms. Both of them try to find a set of forwarding nodes, which can work as virtual backbone of the wireless network.

Guha and Khuller firstly proposed a 2-phase centralized greedy algorithm to construct CDS in general graphs [8]. At first, the algorithm finds a dominating set and then connects the dominating set. Many distributed approximation algorithms have been proposed in the literature for the minimum CDS (MCDS) [9-12]. These algorithms which obtain better time and message complexities, and smaller CDS size.

However, these above methods ignore two natural issues of MANET: the limited energy and the mobility of nodes. Some related works have been proposed to solve one problem. On the limited energy, many papers tries to construct a CDS by taking energy as an import factor [13-1819]. A method of calculating the power-aware that connects the dominating set [13] was proposed by $\mathrm{Wu}$ et al. Further, an algorithm was proposed by Rai etc. to construct a power aware minimum connected dominating set, which goes three phases.

For the mobility of the nodes, several works took the mobility into consideration to construct CDS [19-21]. An algorithm [19] proposed by Wang et al. took node stability into consideration, and it could construct CDS quickly. Also, Sheu et al. proposed an approach [21] to calculate stable CDSs based on link stability.

Therefore, it is observed that the energy and movement of nodes exert direct impacts to construct a CDS. As motivated by the ideas, we propose a novel approach to construct CDS in MANET in terms of three factors of energy, mobility and degree of the nodes.

\section{Constructing Cgreedy-wMCDS}

In this Section, a two-phase algorithm is proposed to construct Cgreedy-wCDS. The first stage is Cgreedy-wDS formation and the second stage is connecting and pruning Cgreedy-wDS.

\subsection{Definition of $S T(u)$}

The current status of node $S T(u)$ is defined as Eq. (1), which considers factors of energy, degree and mobility of node $u$.

$$
S T(u)= \begin{cases}\frac{E_{s}}{V_{s}} \cdot|\operatorname{deg} r e e(u)|, & \text { if } V_{s}>0 \\ E_{s} \cdot|\operatorname{deg} r e e(u)|, & \text { otherwise }\end{cases}
$$

Definition 1 (the mobility of a node) $V$ considers its neighbor set. Its mobility factors can be valued as the percentage of neighbors which remains the same between $t$ time and $t+1$ time. With the symmetric difference $V$, the algorithm can select more stable nodes as dominate node. 


$$
V_{s}=\frac{N^{t+1}(u) \backslash N^{t}(u)}{N^{t+1}(u) \cup N^{t}(u)}
$$

Where, the form of $A \backslash B$ denotes the symmetric difference between $A$ and $B$.

Definition 2 (the energy metric of a node) $E s$ is defined as Eq. (3). The metrics $E s$ help algorithm to select energy-rich nodes.

$$
E_{s}=\frac{E_{r m}(u)}{E_{\text {in }}(u)}
$$

\subsection{Cgreedy-Weds Formation}

Each mobile node transmits a hello message to its neighbors. $\langle S T(u), N(u)\rangle$ is included in the message. A node gets its one and two hop neighbor information when it receives this

\begin{tabular}{|c|c|}
\hline $\begin{array}{l}\text { Input: a connected graph; every } \\
\text { node in knows it's one hop and } \\
\text { two hop neighbors. } \\
\text { Result: is marked in ,or, } \mathrm{D}=\varnothing \text {; } \\
\text { For each node in } \\
\text { While (maximum nodal degree } \\
\text { in }>0 \text { ) } \\
\text { Do } \\
\text { For each node in } \\
\text { If the value of is the } \\
\text { maximum in its one hop neighbor } \\
\text { and two hop neighbors } \\
\text {. } \\
\text { incident on them form } \\
\quad \text { Update the degree of the } \\
\text { remaining nodes in } \\
\quad / * \text { In case of a tie, a node } \\
\text { which hasd higher degree is } \\
\text { marked withinin. If still a tie, the } \\
\text { node with more energy is given } \\
\text { preference. } \\
\text { End if } \\
\text { End for }\end{array}$ & $\begin{array}{l}\text { Input: each node in knows its one hop neighbor and two } \\
\text { hop neighbors. } \\
\text { Output: CDS: dominate set formed by black nodes } \\
\text { All nodes and nodes form separate components. } \\
\text { If all black nodes are not in the same component } \\
\text { Select the connector to connect the components } \\
\text { which cover, according to the rules as follows : } \\
\text { Dominatee with the greatest value ofis selected to } \\
\text { connect components which covers. } \\
\text { If is result in a tie } \\
\text { Dominatee with the higher degree is preferred. } \\
\text { End if } \\
\text { If the degree and result in a tie } \\
\text { Dominatee with more energy is preferred. } \\
\text { End if } \\
\text { End if } \\
\text { For each node } \\
\text { If any color of is of } \\
\text { CDS }=C D S-\{w\}\end{array}$ \\
\hline
\end{tabular}
message from its neighbors.

\section{Algorithm 1 :Forming Cgreedy-D Algorithm 2 :Forming Cgreedy-wMCDS}

The process which forms a Cgreedy-wDS is described in Algorithm 1. Initially, all nodes in network are marked in WHITE color. If any node $u$ in $V-D$ has the highest $S T(u)$ value in its 1-hop and 2-hop neighborhood. $u$ is selected as the backbone node of the network. Then delete all the adjacent nodes of $u$ and edges incident on them from $G$, which is repeated if the maximum nodal degree in $G>0$. Each undeleted node in $G$ excepting the dominators in $D$ is added to the DS if its degree is zero, and its color is changed to GRAY.

Cgreedy-Wcds can be obtained by Algorithm 2. The main idea of Algorithm 2 is as follows: all dominators and virtual-dominators form the separate component. $u$ as connector which can adjacent to the maximum number of separate components is selected.

\subsection{Theoretical Analysis}

Theorem 1: connect dominate set can be constructed by the above algorithm. 
Proof: our algorithm has three stages. The dominate set is constructed after the first phase that all the dominatees are the neighbors of Black node. In the second phase, connect all the black nodes until all black nodes are in the same component. Finally, some dominate nodes are deleted, but this operation did not break the connectivity of the Dominate set. Those nodes, as neighbors of BLACK node are deleted. So our algorithm is correct.

Theorem 2: the time complexity of our algorithm is $O(D)$, where $D$ is the network diameter.

Proof: in our method, all nodes need to maintain its two-hop neighborhood information. Every node sends a hello message to its neighbors at intervals. Each node can update its one hop and two hop neighborhood information in time. The distributed impletion of our algorithm will require at most $O(n)$. The two phases in Cgreedy-wMCDS are based on the selection of multiple leaders. The time complexity of our algorithm is $O(D)$, where $D$ is the network diameter.

\subsection{Routing on Cgreedy-wMCDS}

The routing protocols can be divided into two groups: flat and hierarchical routing protocols, according to the logic of the network structure. In a flat routing protocol, every node is set to equal status and discovers a route with a broadcasting scheme. In this case, every node has to repeat sending or receiving broadcast signals [24]. In turn, every receiving node broadcasts the RREQ packet until the packet reaches the destination. Finally, the destination node will send the route reply message (RREP) to the source after receiving the RREQ packet. However, this protocol leads to an issue of broadcast storm, especially in MANET with large scale. To solve the problem more efficiently, we implemented a route discovery process in AODV with the proposed Cgreedy-MCDS. When a Cgreedy-CDS node receives a RREQ packet, it broadcasts the packet. But the nodes out of Cgreedy-CDS only receive the packet. So, the number of RREQ packet is reduced and the network congestion may be avoided to a large extent.

\section{Simulation Results}

In the section, some simulations of the proposed Cgreegy-wMCDS show better efficiency in comparison with the existing previous approaches. Our algorithm is written with matlab 7.0, and 90 trials simulated experiments are repeated with different network sizes and speeds of mobile nodes. The parameters in our experiments are listed as follows in TABLE 1.

\subsection{Average CDS Size}

The simulation result of the average size of CDS with varying network sizes is shown in Figure 2. It is apparent that the CDS size of our method is smaller than Wu-CDS. And the 


\begin{tabular}{|ll|ll|}
\hline \multicolumn{2}{|c|}{ TABLE I. } & TABLE II. & value \\
\hline TABLE III. & Area size & TABLE IV. $\quad 1000 * 1000 \mathrm{~m} 2$ \\
\hline TABLE V. & Traffic type & TABLE VI. $\quad$ Constant Bit Rate(CBR) \\
\hline TABLE VII. & Packet size & TABLE VIII. & 512 bytes \\
\hline TABLE IX. & MAC protocol & TABLE X. $\quad$ IEEE 802.11 \\
\hline TABLE XI. & Transmission range & TABLE XII. $\quad 250 \mathrm{~m}$ \\
\hline TABLE XIII. & Bandwidth & TABLE XIV. & $2 \mathrm{Mbps}$ \\
\hline TABLE XV. & Queue size & TABLE XVI. & 50 packets \\
\hline TABLE XVII. & Mobility Model & TABLE XVIII. & Random Waypoint Model \\
\hline TABLE XIX. & transmission power & TABLE XX. & $0.667 \mathrm{w}$ \\
\hline TABLE XXI. & Receiving power & TABLE XXII. & $0.365 \mathrm{~W}$ \\
\hline TABLE XXIII. & Idle power & TABLE XXIV. & $0.1 \mathrm{w}$ \\
\hline TABLE XXV. & Maximum speed & TABLE XXVI. & $5,10,15,20,25 \mathrm{~m} / \mathrm{s}$ \\
\hline
\end{tabular}

Table 1: Simulation parameters

average CDS size of DAS-CDS [15] is also smaller than our method because DAS-CDS constructed a connected dominating set through multi-leader initiated degree-based method.

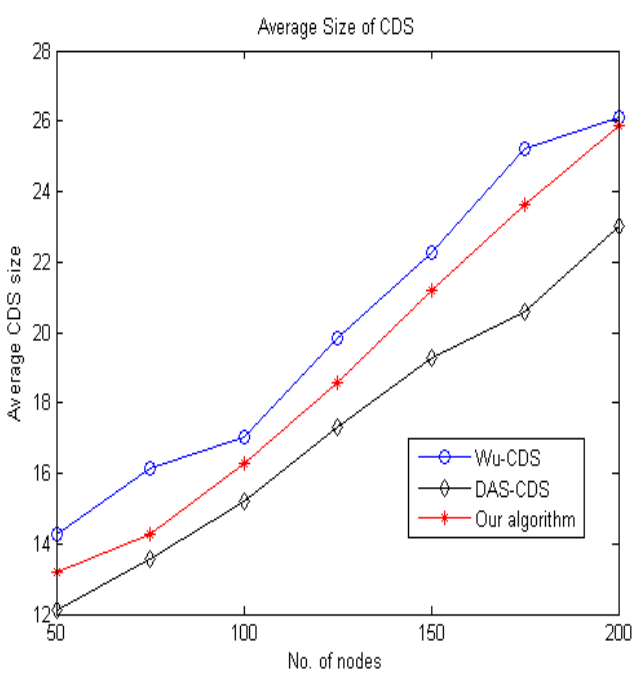

Figure 2: Average Size of CDS

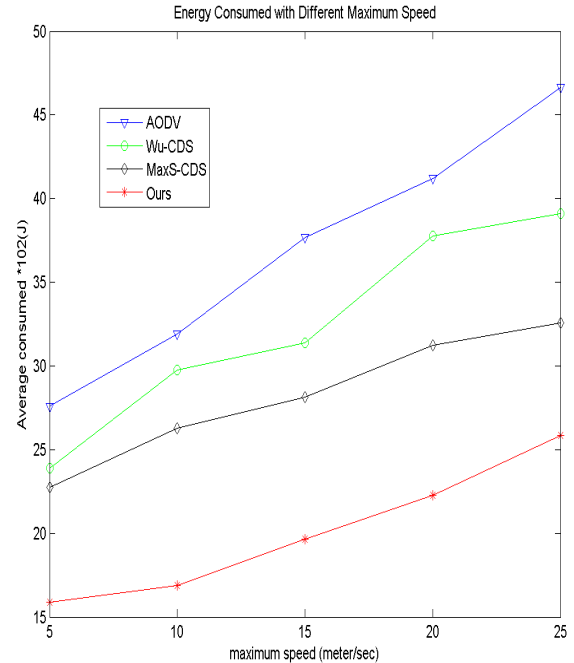

Figure 3: Average Energy Consumed

\subsection{Average Energy Consumed}

Figure 3 depicts the total energy consumed with different maximum speeds with increasing number of nodes. Our algorithm prefers to select more stable nodes. The total energy is smaller because the routes are highly prone to breakage when the host speed increases.

\subsection{Average Life Time of Network}

The lifetime of MANET with increasing number of nodes is showed in Figure 4. Obviously, this result illustrates that nodes with more residual energy are selected as backbone nodesdue to the restriction of the confined energy of the batteries. At same time, the proposed method selects smaller nodes as backbone. Thus, the volume of the messages transmitted in the network will be reduced. As a consequence, the life time of the network can be prolonged significantly. 


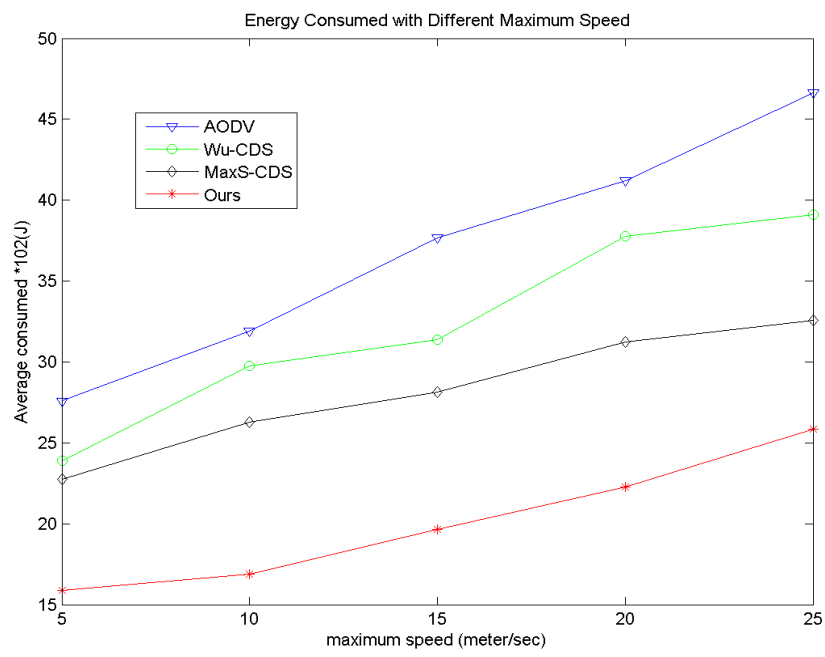

Figure 4 : Average Life Time of Network

\section{Conclusion}

In the paper, a new approach of constructing a Cgreedy-wMCDS was proposed to maximize the networks lifetime by constructing a CDS in MANET with a new greedy distributed algorithm. In the proposed method, three factors of energy, movements and degree of nodes are considered. The simulation results show that it is better than other methods.

\section{References}

[1] Broch J, Maltz D A, Johnson D B, et al. A performance comparison of multi-hop wireless ad hoc network routing protocols $[\mathrm{C}] / /$ Proceedings of the 4 th annual ACM/IEEE international conference on Mobile computing and networking. ACM, 1998: 85-97.

[2] Macker J. Mobile ad hoc networking (MANET): Routing protocol performance issues and evaluation considerations[J]. 1999.

[3] B. Clark, C. Colborn, and D. Johnson, "Unit disk graphs, " Discrete mathematics, vol. 86, no. 1, Aug. 1990 , pp.165-177.

[4] Bai F, Helmy A (2004) A survey of mobility modeling and analysis in wireless ad hoc networks. In: Wireless ad hoc and sensor networks. Kluwer Academic, Dordrecht

[5] C. Zheng. S.-X. Sun, and T.-Y. Huang, "Constructing Distributed Connected Dominating Sets in Wireless Ad Hoc and Sensor Networks,” J. Softw., vol. 22, no. 5, Jun. 2011, pp. 1053-1066.

[6] Wu, Jie, and Li Hai-lan. "On calculating connected dominating set for efficient routing in ad hoc wireless networks." Proceedings of the 3rd international workshop on Discrete algorithms and methods for mobile computing and communications. ACM, 1999.

[7] Ephremides A, Wieselthier J E, Baker D J. A design concept for reliable mobile radio networks with frequency hopping signaling[J]. Proceedings of the IEEE, 1987, 75(1): 56-73.

[8] Guha S, Khuller S. Approximation algorithms for connected dominating sets[J]. Algorithmica, 1998, 20(4): 374-387.

[9] V. Bharghavan and B. Das, "Routing in Ad Hoc Networks Using Minimum Connected Dominating Sets”, International Conference on Communications'97, Montreal, Canada. June 1997. 
[10] J. Wu and H.L. Li, “On calculating connected dominating set for efficient routing in ad hoc wireless networks", Proceedings of the 3rd ACM international workshop on Discrete algorithms and methods for mobile computing and communications, 1999, Pages 7-14.

[11] I. Stojmenovic, M. Seddigh, J. Zunic, “Dominating sets and neighborelimination based broadcasting algorithms in wireless networks", Proc.IEEE Hawaii Int. Conf. on System Sciences, January 2001

[12] Alzoubi K M, Wan P J, Frieder O. New distributed algorithm for connected dominating set in wireless ad hoc networks[C]//System Sciences, 2002. HICSS. Proceedings of the 35 th Annual Hawaii International Conference on. IEEE, 2002: 3849-3855.

[13] . Wu, J., Dai, F., Gao, M., \&Stojmenovic, I. (2002). On calculating power-aware connected dominating sets for efficient routing in ad hoc wireless networks. IEEE/KICS Journal of Communications and Networks, 4, 59-70.

[14] Yu,R., Wang, X., \& Das, K. (2009). EEDTC: Energy efficientdominating tree construction in multihop wireless networks. Pervasive and Mobile Computing, 5, 318-333.

[15] Rai, M., Verna, S., \&Tapaswi, S. (2009). A power aware minimum connected dominating set for wireless sensor networks. Journal of Networks, 6(6), 511-519.

[16] . Kim, B., Yang, J., Zhou, D., \& Sun, M. (2005). Energy aware conneced dominating set construction in mobile ad hoc networks. InAuburn University Technical Report CSSE05-07.

[17] Shukla, K. K., \&Sah, S. (2013). Construction and maintenance of virtual backbone in wireless networks. Wireless Networks. doi:10. 1007/s11276-012-0512-6.

[18] Torkestani J A. An energy-efficient topology construction algorithm for wireless sensor networks[J]. Computer Networks, 2013, 57(7): 1714-1725

[19] Wang, F., Min, M., Li, Y., \& Du, D. (2005). On the construction of stable virtual backbones in Mobile ad hoc networks. In Proceedings for the IEEE international performance computing and communicaions conference

[20] Sheu, P. R., Tsai, H. Y., Lee, Y. P., \& Cheng, J. Y. (2009). On calculating stable connected dominating sets based on link stability for mobile ad hoc networks. Tamkang Journal of Science and Engieering, 12(4), 417-428.18. Leu, S., \& Chang, R. (2012).

[21] A weight-value algorithm forfinding connected dominating set in a MANET. Journal of Network and Computer Applications, 35, 1615-1619

[22] Shukla, K. K., and S. Sah. "Construction and maintenance of virtual backbone in wireless networks." Wireless networks 19.5 2013: 969-984. 